\title{
A method to determine the radial compliance of porcine coro- nary arteries ex vivo via optical coherence tomography
}

Christoph Brandt-Wunderlich, Institute for ImplantTechnology and Biomaterials e.V, Rostock-Warnemuende, Germany, e-Mail: christoph.brandt-wunderlich@uni-rostock.de

Franziska Bonin, Institute for Biomedical Engineering, Rostock University Medical Center, RostockWarnemuende, Germany, e-Mail: franziska.bonin@uni-rostock.de

Wolfram Schmidt, Institute for Biomedical Engineering, Rostock University Medical Center, RostockWarnemuende, Germany, e-Mail: wolfram.schmidt@uni-rostock.de

Swen Großmann, Institute for ImplantTechnology and Biomaterials e.V, Rostock-Warnemuende, Germany, e-Mail: swen.grossmann@uni-rostock.de

Michael Stiehm, Institute for ImplantTechnology and Biomaterials e.V, Rostock-Warnemuende, Germany, e-Mail: michael.stiehm@uni-rostock.de

Alper Öner, Heart Center/Department of Cardiology, Rostock University Medical Center, Rostock, Germany, e-Mail: alper.oener@med.uni-rostock.de

Niels Grabow, Institute for Biomedical Engineering, Rostock University Medical Center, RostockWarnemuende, Germany, e-Mail: niels.grabow@uni-rostock.de

Klaus-Peter Schmitz, Institute for ImplantTechnology and Biomaterials e.V and Institute for Biomedical Engineering, Rostock University Medical Center, Rostock-Warnemuende, Germany, e-Mail: klauspeter.schmitz@uni-rostock.de

Stefan Siewert, Institute for ImplantTechnology and Biomaterials e.V, Rostock-Warnemuende, Germany, e-Mail: stefan.siewert@uni-rostock.de

\section{Introduction}

Since the first application in medicine in 1991 optical coherence tomography (OCT) was introduced as imaging method for various applications in ophthalmology, oncology and cardiology. For intravascular imaging the rotating optic in the distal tip of a special OCT catheter is used to obtain cross sectional images of the artery. Due to the high spatial resolution, OCT is suitable for precise measurements of the intraluminal diameter of coronary arteries. The aim of the current study was to develop a test method for determination of the compliance of porcine coronary arteries.

\section{Methods}

A porcine heart was prepared for ex vivo OCT measurements within the left anterior descending coronary artery (LAD). A constant media flow was applied to the LAD resulting in a specific intra-arterial pressure. Diameter measurements after OCT imaging were performed during consecutively pressurization of the LAD from 40 to $200 \mathrm{mmHg}$ in a physiological environment. The arterial compliance was determined for physiological $(120 / 80 \mathrm{mmHg})$ and a hypertension pressure range $(160 / 100 \mathrm{mmHg})$. For OCT measurements a Dragonfly OPTIS Imaging Catheter (Abbott Medical) in combination with the ILUMIEN OPTIS PCI Optimization system (St. Jude Medical) was used.

\section{Results}

Diameter measurements and pressure measurements could be performed with a high accuracy (diametral resolution $\pm 15 \mu \mathrm{m}$, pressure measurement uncertainty $3 \%$ within the range of 0 to $258 \mathrm{mmHg}$ ). A radial compliance of $5.7-6.7 \% / 100 \mathrm{mmHg}$ for the distal segment, $3.8-8.5 \% / 100 \mathrm{mmHg}$ for the middle segment and $4.7-8.6 \% / 100 \mathrm{mmHg}$ for the proximal segment of the LAD was measured, depending on the considered pressure range.

\section{Conclusion}

The test setup used for determination of the arterial compliance ex vivo showed very reproducible results und therefore is suitable to generate clinical relevant data. Further studies should include a various number of porcine hearts and coronary arteries, respectively to gain statistical significance of the test results. 
Abstracts - BMT 2020 - Leipzig, September 29 - October 1·DOI 10.1515/bmt-2020-6@@@

Biomed. Eng.-Biomed. Tech. 2020; 65(s1): S@@@-S@@@•@ by Walter de Gruyter•Berlin•Boston 


\section{EIT based intrathoracic pulsatile impedance measurements during apnea: a case study}

Sabine Krueger-Ziolek, Institute of Technical Medicine (ITeM), Furtwangen University, Villingen-Schwenningen, Germany, krue@hs-furtwangen.de

András Lovas, Department of Anaesthesiology and Intensive Therapy, University of Szeged, Szeged, Hungary, lovas.andras@med.u-szeged.hu

Fatime Hawchar, Department of Anaesthesiology and Intensive Therapy, University of Szeged, Szeged, Hungary, hawchar.fatime@med.u-szeged.hu

Bernhard Laufer, Institute of Technical Medicine (ITeM), Furtwangen University, Villingen-Schwenningen, Germany, lab@hs-furtwangen.de

Knut Moeller, Institute of Technical Medicine (ITeM), Furtwangen University, Villingen-Schwenningen, Germany, moe@hs-furtwangen.de

For the Digital Clones in Personalized Medicine (DCPM) study team: Balázs Benyó ${ }^{1}$, Geoff Chase ${ }^{2}$, Thomas Desaive ${ }^{3}$, Knut Moeller ${ }^{4}$

${ }^{1}$ Department of Control Engineering and Information, Budapest University of Technology and Economics, Budapest, Hungary.

${ }^{2}$ Centre for Bioengineering, University of Canterbury, Christchurch, New Zealand.

${ }^{3}$ GIGA Cardiovascular Science, University of Liege, Liege, Belgium.

${ }^{4}$ Institute of Technical Medicine (ITeM), Furtwangen University, Villingen-Schwenningen, Germany.

\section{Introduction}

Intrathoracic ventilation related and pulsatile (perfusion) impedance changes can be measured by the non-invasive and radiation-free imaging method Electrical Impedance Tomography (EIT). Ventilation monitoring is still the key research area in EIT, whereby perfusion monitoring gain more and more in interest. However, there are still many unknown influencing factors concerning pulsatile impedance measurements which have to be investigated.

\section{Methods}

Hence, in this observational case study the impact of prolonged apnea periods on pulsatile impedance changes was examined in a patient with suspected brain death undergoing several apnea tests. In addition, the correlation between changes in pulsatile impedance and certain blood gas parameters (carbon dioxide partial pressure, pCO2; oxygen partial pressure, $\mathrm{pO} 2$; pH; bicarbonate, $\mathrm{HCO} 3-)$ were explored.

\section{Results}

Results show that the pulsatile impedance signal changes over time during apnea. An increase in the area under the curve (Mean $\mathrm{AuC}$ ) and the maximum amplitude (Mean Max) of heart beat associated impedance signals was observed (Mean AuC: up to $65 \%$; Mean Max: up to $57 \%$ ). Furthermore, a positive correlation between the increase in impedance and pCO2 and $\mathrm{HCO} 3$ - was assessed (both: up to 0.99), whereas pO2 and pH show a negative correlation (both: up to -0.99$)$.

\section{Conclusion}

These preliminary results indicate that pulsatile EIT monitoring may be applied to get additional information regarding cardio-pulmonary interactions sustaining diagnosis or treatment of lung diseases. 


\section{Automated Cephalometric Landmark Localization using a Coupled Shape Model}

Andreas Wirtz, aVisual Healthcare Technologies, Fraunhofer IGD, Darmstadt, Germany, e-mail: andre-

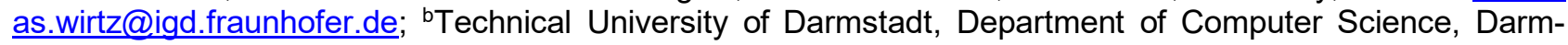
stadt, Germany

Julian Lam, Visual Healthcare Technologies, Fraunhofer IGD, Darmstadt, Germany

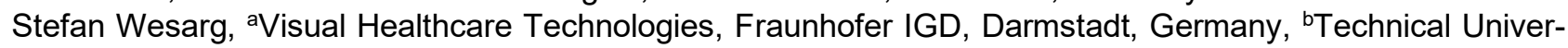
sity of Darmstadt, Department of Computer Science, Darmstadt, Germany

\section{Introduction}

In orthodontics, the cephalometric analysis is used to study the soft tissue-, dental- and bony structures of a patient. In clinical practice, the landmarks used for this analysis need to be manually traced which is a time consuming and subjective task. Automated landmark detection would be beneficial but is difficult to achieve with high precision and success rate.

\section{Methods}

We present an approach that combines a 2-D coupled shape model for predicting the landmark locations of 19 landmarks with a Hough Forest to refine each individual location. In contract to other methods, we not only use the spatial relation between landmarks but also include their spatial relation to additional structures like skull or skin. To achieve this, we have extended our coupled shape model framework to include single point structures like landmarks. This way, the search space for each landmark can be narrowed down and the final position in a small region around the predicted location can be determined based on its local intensity appearance. The coupled shape model and Hough Forests were trained on 50 and 120 images, respectively.

\section{Results}

Performance has been evaluated on 250 test images which have not been used during model training or development. The achieved localization results vary between landmarks depending on how well defined their local intensity appearance is. Overall, the presented approach achieves a competitive performance with a successful detection rate of $70.24 \%, 79.14 \%, 83.97 \%$ and $89.82 \%$ for accuracy ranges of $2.0,2.5,3.0$ and $4.0 \mathrm{~mm}$, respectively.

\section{Conclusion}

In conclusion, exploiting not only the spatial relation between landmarks but also the relation to other anatomical structures like the skull or the skin via a coupled shape model proves effective for predicting the landmarks locations. Location refinement based on local intensity appearance using a Hough Forest works well for most landmarks. 


\title{
Estimating regional pulmonary blood flow in EIT with regularized deconvolution with a Tikhonov regularization
}

\author{
Xenia Augustin, Institute of Biomedical Engineering, KIT, Fritz-Haber-Weg 1, Karlsruhe, Germany, e-mail: \\ publications@ibt.kit.edu \\ Michael Kircher, Institute of Biomedical Engineering, KIT, Karlsruhe, Germany, e-mail: Mi- \\ chael.kircher@kit.edu \\ Olaf Dössel, Institute of Biomedical Engineering, KIT, Karlsruhe, Germany, e-mail: olaf.doessel@kit.edu \\ Birgit Stender, Drägerwerk AG \& Co. KGaA, Lübeck, e-mail: birgit.stender@draeger.com \\ Thomas Bluth, Pulmonary Engineering Group Dresden, Dep. of Anesthesiology and Intensive Care Medi- \\ cine, University Hospital \& Technische Universität, Dresden, Germany, e-mail: Thomas.Bluth@uniklinikum- \\ dresden.de \\ Marcelo Gama de Abreu, Pulmonary Engineering Group Dresden, Dep. of Anesthesiology and Intensive \\ Care Medicine, University Hospital \& Technische Universität, Dresden, Germany, e-mail: \\ mgabreu@uniklinikum-dresden.de
}

\section{Introduction}

Electrical Impedance Tomography (EIT) is a clinically used tool for bed-side monitoring of ventilation. Previous work also showed a high potential for lung perfusion monitoring with indicator-enhanced EIT. However, many research questions have yet to be answered before it can be broadly applied in clinical everyday life. The goal of this work is to evaluate a new method to improve EIT perfusion measurements.

\section{Methods}

Pulmonary hemodynamic transfer functions were estimated using regularized deconvolution with Tikhonov regularization to estimate spatial perfusion parameters. The pulmonary blood flow rBF was determined from the transfer function. The resulting EIT images were compared with PET Scans.

\section{Results}

The final comparison between EIT images and PET scans showed a median correlation of 0.935 for the images which were reconstructed using the regularized deconvolution. In comparison the previously used maximum

slope method led to a median correlation of 0.912 .

\section{Conclusion}

The quantitative comparison of EIT images and PET scans showed that the methods investigated led to a good correlation of EIT images and PET scans. A clear improvement of the spatial similarity to PET using the regularized deconvolution reconstruction could not be observed. 
L. H. Kohler, H. Köhler, S. Kohler, S. Langer, R. Nuwayhid, I. Gockel, N. Spindler, G. Osterhoff

Hyperspectral imaging (HSI) as a new diagnostic tool in free flap monitoring for soft tissue reconstruction

\section{OBJECTIVES:}

Free flap surgery is a routine procedure in reconstructive surgery of soft tissue defects after trauma or infection. A soft tissue flap is harvested from a healthy body region and transplanted into the defect. Complications of perfusion after free tissue transplantation are common and often require revision surgery and total removal of the flap. Aim of any postoperative monitoring is the detection of vessel perfusion disorders as soon as possible. The clinical gold standards are clinical examination and vascular Doppler and have been established centuries ago. No innovative processes have so far been able to supplement or even replace these established diagnostic tools. We present the technique of hyperspectral imaging (HSI) as a new tool in postoperative flap monitoring to improve sensitivity compared to established tools.

\section{METHODS:}

The HSI camera records light spectra from 500 to $1000 \mathrm{~nm}$ and creates a three-dimensional dataset called Hypercube, with two spatial dimensions and one spectral dimension. The resulting data provides diagnostic information on tissue morphology, composition, and physiology. These parameters contain tissue oxygenation (StO2), perfusion (NIR), tissue hemoglobin (THI), and tissue water index (TWI) in the range from 0 to 100.

We performed an open-label, single-arm and non-randomized intervention clinical trial including 22 patients. 3 HSI images were taken ( $\mathrm{t} 1=0 \mathrm{~h}, \mathrm{t} 2=16-28 \mathrm{~h}$, and $\mathrm{t} 3=39-77 \mathrm{~h}$ after surgery) and analyzed with TIVITA ${ }^{\oplus}$ Suite.

\section{RESULTS:}

Complete flap revision was performed in $n=4$ and partial revision in $n=2$ patients. All flaps with a StO2 and NIR PI below 40 at $\mathrm{t} 2$ were revised later on. All viable flaps showed StO2 or NIR PI above 40 at t2. Significant differences between viable ( $\mathrm{StO}=49 ; \mathrm{NIR} \mathrm{PI}=45 ; \mathrm{THI}=16 ; \mathrm{TWI}=56)$ and revised flaps (StO2=28 $[\mathrm{p}<0.001]$; NIR $\mathrm{Pl}=26[\mathrm{p}=0.002] ; \mathrm{THI}=56[\mathrm{p}=0.002] ; \mathrm{TWI}=47[\mathrm{p}=0.045])$ were present in all tissue parameters at $\mathrm{t} 2$. These differences were even more significant ( $p<0.0001$ for all tissue parameters) at $\mathrm{t} 3$.

\section{CONCLUSION:}

Based on this data, HSI seems to be a valuable diagnostic tool to monitor perfusion and oxygenation of transplanted free soft tissue flaps in reconstructive surgery. The technique may detect complications at a very early stage where the surgical team might be able to react before the transplant is lost. Different parameters may also allow differentiating between issues based on arterial inflow and venous outflow. With more patients planned to be included, the goal is to provide critical values and thresholds for postoperative HSI monitoring that would guide surgeons in their decision and optimize patients' outcomes in free flap surgery. 